\title{
GADZOOKS! (SuperK-Gd): status and physics potential
}

\author{
Pablo Fernández* \\ for the Super-Kamiokande Collaboration \\ E-mail: pablo.fernandezmeuam.es
}

The GADZOOKS! project was the proposed upgrade of the Super-Kamiokande (SK) detector in order to enable it to efficiently detect thermal neutrons by adding gadolinium $(\mathrm{Gd})$. It was approved by the Super-Kamiokande Collaboration in its General Meeting of June 2015 and renamed SuperK-Gd.

Inverse beta decay reactions, as well as charged current quasi-elastic (CCQE) scattering of low energy anti-neutrinos (up to a few hundreds of $\mathrm{MeV}$ ) in SK, produce one positron and one neutron in the final state. The neutron thermalises and is captured by gadolinium. The capture is followed by a $8 \mathrm{MeV}$ gamma cascade. The observation of this cascade would mean that SK could identify these reactions as genuine with very high efficiency $(>80 \%)$. Using this technique, SuperK-Gd will enable Super-Kamiokande - and water Cherenkov detectors in general - to pursue a wealth of physics which is currently inaccessible due to backgrounds. Probably, the most important will be the first observation of the diffuse supernova neutrino background: Super-Kamiokande enriched with Gd can discover it after few years of running. In addition, Gd-loading has benefits for other physics currently shadowed by backgrounds, such as subtle features of supernova bursts, pre-supernovae and reactor antineutrinos. Gd would also provide significant improvements for atmospheric and accelerator neutrino analysis and proton decay searches.

The main R\&D program towards SuperK-Gd has been EGADS: a 200 ton, fully instrumented tank built in a new cavern in the Kamioka mine. EGADS incorporated all the necessary subsystems to make SuperK-Gd a reality.

This contribution will describe the main physics topics and the most important results from the EGADS and other R\&D programs towards SuperK-Gd.

The 34th International Cosmic Ray Conference

30 July- 6 August, 2015

The Hague, The Netherlands

\footnotetext{
*Speaker.
} 


\section{SuperK-Gd general description}

Super-Kamiokande (SK) is a 50,000 ton water Cherenkov detector located in the Kamioka mine under $1000 \mathrm{~m}$ of rock, which began the data taking in 1996. The detector is divided into inner and outer detector, the former is used for physics measurements and instrumented with 11146 PMTs of 20 inches, while the latter is used as a veto to reduce background and is instrumented with 1885 PMTs of 8 inches. The typical volume used for physics measurements is 22,500 tons, 2 $\mathrm{m}$ away from the inner detector wall.

By dissolving a $0.2 \%$ by mass of $\mathrm{Gd}$, in form of a salt $\left(\mathrm{Gd}_{2}\left(\mathrm{SO}_{4}\right)_{3}\right)$, into the SK water [1], the majority of final state neutrons produced in the interactions will thermalised and be captured by $\mathrm{Gd}$ and detected through the $8 \mathrm{MeV} \gamma$ ray cascade from its de-excitation. At this concentration, the time elapsed after the prompt signal (charged particle from neutrino interaction) is about $35 \mu \mathrm{s}(\sim 15 \mu$ s for neutron thermalisation and $\sim 20 \mu$ s for the neutron to be absorbed by Gd).This method is around 5 times more efficient and much more pure than the current technique, which tags some of the final state neutrons by detecting the $2.2 \mathrm{MeV}$ photon emitted by the deuteron produced when a neutron is captured by the hydrogen present in the water.

This efficient neutron tagging provides a very trustable

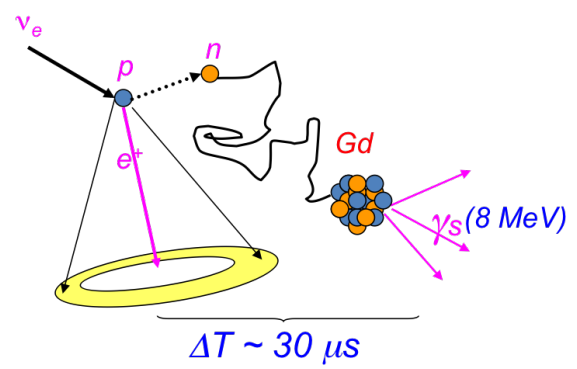

Figure 1: Inverse $\beta$-decay in SuperK-Gd way to distinguish low energy antineutrinos from neutrinos through inverse $\beta$-decay.

\section{Expected physics}

SuperK-Gd will improve some of the already existing measurements from SK and unveil other new measurements. The advantages of Gd-neutron tagging can be seen at low and high energies.

Low energy: One of our main goals for this upgrade is to be able to first detect Diffuse Supernova Neutrino Background (DSNB). This measurement will provide very important information, like the mean energy spectrum core collapse supernovae and the star formation rate of the universe, and it is currently dominated by spallation background. If a supernova occurs close enough, providing much information about early stages of the core-collapse process, its spectrum and time profile for neutrinos and antineutrinos independently. In addition, if the supernova is less than $1 \mathrm{kpc}$ away SuperK-Gd will be able to detect the Si burning phase days before to the core-collapse through the monotonically increasing rate of low energy antineutrinos during this stage.

SuperK-Gd will be able to get rid off most of the backgrounds for reactor antineutrinos below 10 $\mathrm{MeV}$, which opens the possibility to do a second analysis for the neutrino oscillation parameters of the solar sector, improving the current sensitivity.

High energy: Neutron tagging by Gd improves the separation between neutrino and antineutrino in the range from $100 \mathrm{MeV}$ to $10 \mathrm{GeV}$, due to the fact that antineutrinos tend to have larger neutron multiplicities in the final state of their interactions. This is useful for long baseline (LB) and atmospheric (ATM) neutrinos and it is expected that the sensitivity for both, CP violating phase and 
mass hierarchy is significantly enhanced. Also for LB and ATM neutrinos, the neutron multiplicity can be used to improve the neutrino energy reconstruction by $40 \%$ because it encloses information about the missed energy in the production of neutral particles.

For proton decay searches, one can remove $83 \%$ of the ATM neutrino background, by requiring the number of neutrons in the final state to be zero.

\section{Results from R\&D program}

EGADS: EGADS (Evaluating Gadolinium's Action on Detector Systems) was a R\&D project for testing the feasibility of adding Gd in water Cherenkov detectors. It consisted of a 200 ton tank instrumented with 227 PMT's of 20" (like those in SK) and other 13 for HK R\&D, a selective filtration system specially designed and developed to deal with Gd-doped water, a pretreatment system for purifying and dissolving $\mathrm{Gd}_{2}\left(\mathrm{SO}_{4}\right)_{3}$, a water transparency monitoring system called UDEAL, an AAS for measuring uniformity of the concentration and a system for removing the $\mathrm{Gd}$ from the water.

Latest results for the water transparency show a very mild effect on it, within the SK range pure water quality. This shows that $\mathrm{Gd}$ will not affect strongly any current data analysis due to light loss (Fig. 2).

Another important measurement is the concentration of the Gd salt in order to check the uniformity along the whole volume and monitor possible Gd losses. This is done with an AAS and measurements show good homogeneity and stability (Fig. 3).

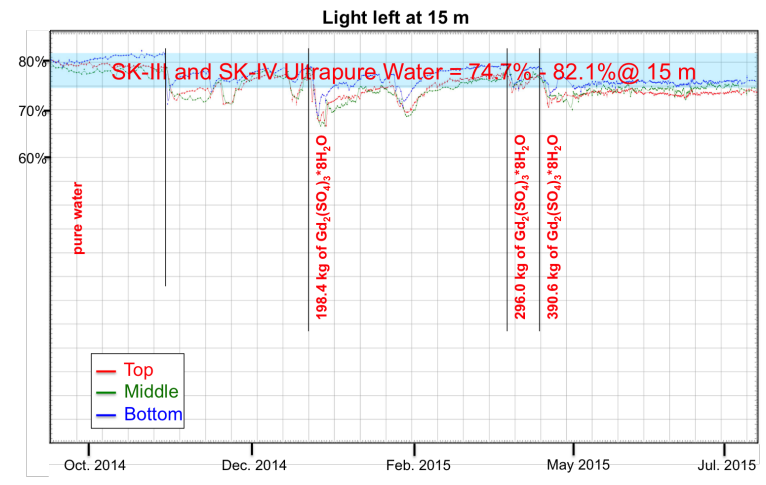

Figure 2: Water transparency measurement by UDEAL (Underground Device Evaluating Attenuation Length).

For checking the Gd-neutron tagging efficiency and its time distribution, an $\mathrm{Am} / \mathrm{Be}$ source is used (Fig. 4).

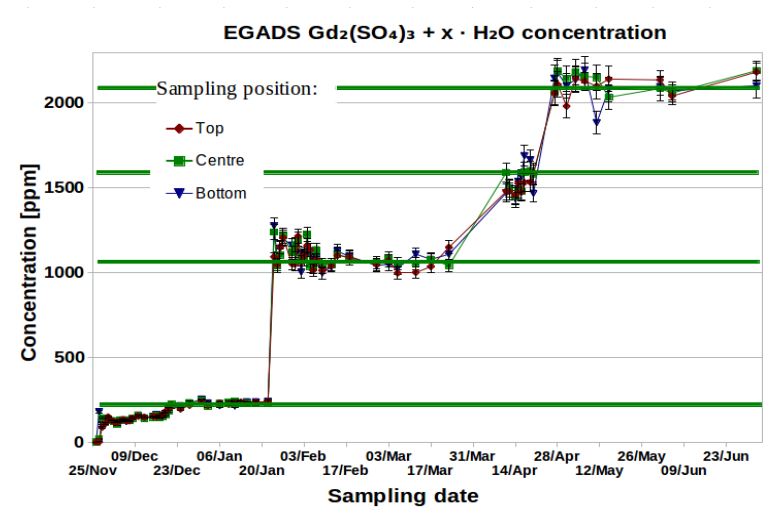

Figure 3: $\mathrm{Gd}_{2}\left(\mathrm{SO}_{4}\right)_{3}$ concentration at instrumented EGADS.

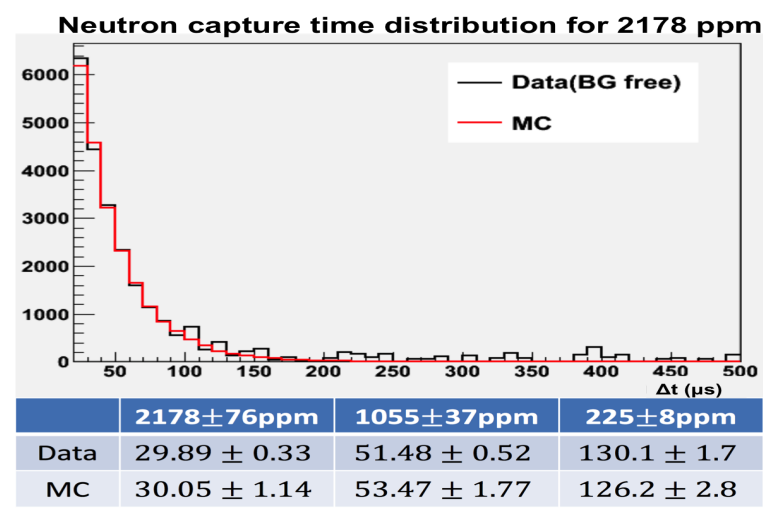

Figure 4: Good agreement in the neutron capture time between data and MC for all Gd concentrations. 
At last, a Rayleigh scattering device was used to judge whether the loss in light is due to absorption or scattering. Results show that this composition is $90 \%$ and $10 \%$ (Fig. 5). This measurement is crucial in order to check the effects of the water transparency loss on all the physics analysis. In particular the neutron detection efficiency for this result becomes $92 \%$, done using multivariate likelihood fed by characteristic variables from the Gd $\gamma$ cascade.

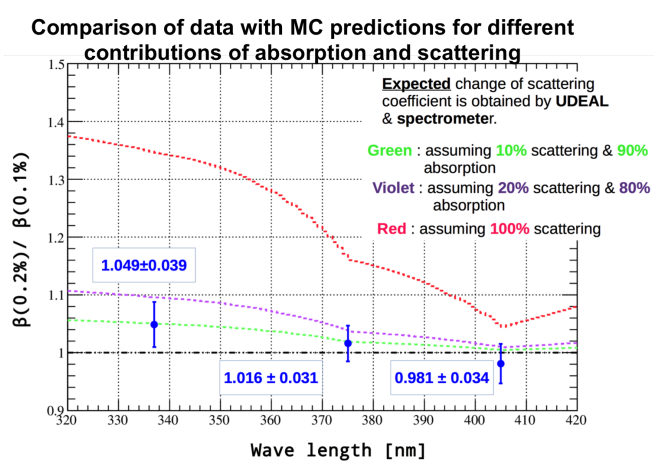

Figure 5: Ratio of scattering coefficient for $0.1 \%$ and $0.2 \% \mathrm{Gd}$ concentration.

Radioactivity from $\mathbf{G d}_{2}\left(\mathrm{SO}_{4}\right)_{3}$ : Since the $\mathrm{Gd}$ compound will be dissolved and uniformly distributed along the whole active volume of the experiment, keeping controlled and as low as possible its radioactive contamination is an important issue. For this purpose an exhaustive campaign is being carried out for measuring the radioactivity of batches from different companies and purification processes. This radioactive contamination basically affects two measurements: DSNB, whose background is under control

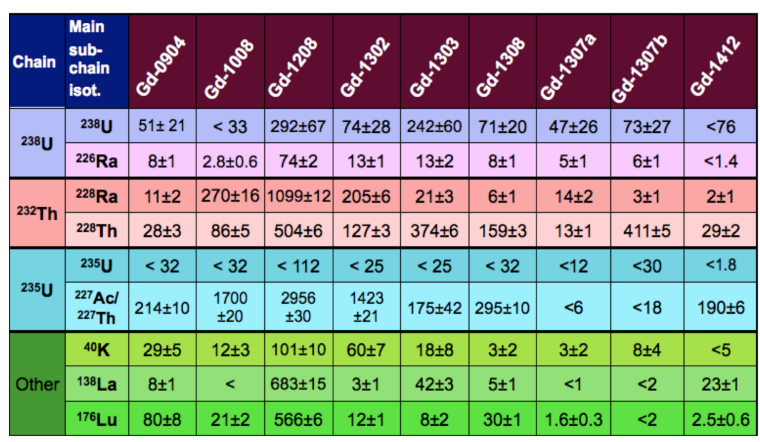

Figure 6: Radiopurity measurements in $\mathrm{mBq} / \mathrm{kg}$ for various $\mathrm{Gd}_{2}\left(\mathrm{SO}_{4}\right)_{3}$ samples at Canfranc Und. Lab. and two orders of magnitude less than the expected signal. For the solar analysis, we need to reduce the amount of $\mathrm{Ra}$ and $\mathrm{Th}$, for which, an additional $\mathrm{R} \& \mathrm{D}$ program is ongoing.

\section{References}

[1] J. F. Beacom, M. R. Vagins, Antineutrino spectroscopy with large water Cherenkov detectors, Phys. Rev. Lett. 93 (2004) 171101. doi:10.1103/PhysRevLett.93.171101.

[2] K. Hirata, et al., Observation of a Neutrino Burst from the Supernova SN 1987a, Phys.Rev.Lett. 58 (1987) 1490-1493. doi:10.1103/PhysRevLett.58.1490.

[3] Y. Fukuda, etal., Constraints on neutrino oscillation parameters from the measurement of day-night solar neutrino fluxes at super-kamiokande, Phys. Rev. Lett. 82 (1999) 1810-1814. doi:10.1103/PhysRevLett.82.1810.

[4] K. Abe, et al., Observation of electron neutrino appearance in a muon neutrino beam.

[5] H. Nishino, et al., Search for nucleon decay into charged antilepton plus meson in Super-Kamiokande I and II, Phys. Rev. D 85 (2012) 112001. doi:10.1103/PhysRevD.85.112001.

[6] K. Bays, oters, Supernova relic neutrino search at super-kamiokande, Phys. Rev. D 85 (2012) 052007. doi:10.1103/PhysRevD.85.052007 